\title{
Assignment of Molecular Structures to the Electrochemical Reduction Products of Diiron Compounds Related to [Fe-Fe] Hydrogenase: A Combined Experimental and Density Functional Theory Study
}

\author{
Stacey J. Borg ${ }^{a}$, Jesse W. Tye ${ }^{b}$, Michael B. Hall ${ }^{b}$ and Stephen P. Best ${ }^{a}$ * \\ ${ }^{a}$ School of Chemistry, University of Melbourne 3010, Victoria, Australia. \\ ${ }^{\mathrm{b}}$ Department of Chemistry, Texas A\&M University, College Station, TX 77843-3255, USA

\section{Supplementary information:}

Fig S1 SEC of 2 in THF/ $\mathrm{TBAClO}_{4}$ under $0.275 \mathrm{MPa} \mathrm{Ar}$

Fig S2 SEC of 2 in THF/ $\mathrm{TBAClO}_{4}$ under $0.275 \mathrm{MPa} \mathrm{CO}$

Fig S3 IR spectra of samples immediately prior to EXAFS sample collection.

Fig S4 EPR spectra of $2 \mathrm{E}$ at room temperature and $100 \mathrm{~K}$

Table S1 Summary of the EXAFS analysis of 2D

Table S2 Summary of the EXAFS analysis of $\mathbf{2 E}$

Table S3 DFT Computed Free Energies (Gas Phase, THF, and $\mathrm{CH}_{3} \mathrm{CN}$ Solution)

Table S4 DFT Computed and Predicted v(CO) Stretching Frequencies

Table S5 DFT optimized structures (in PDB format) 


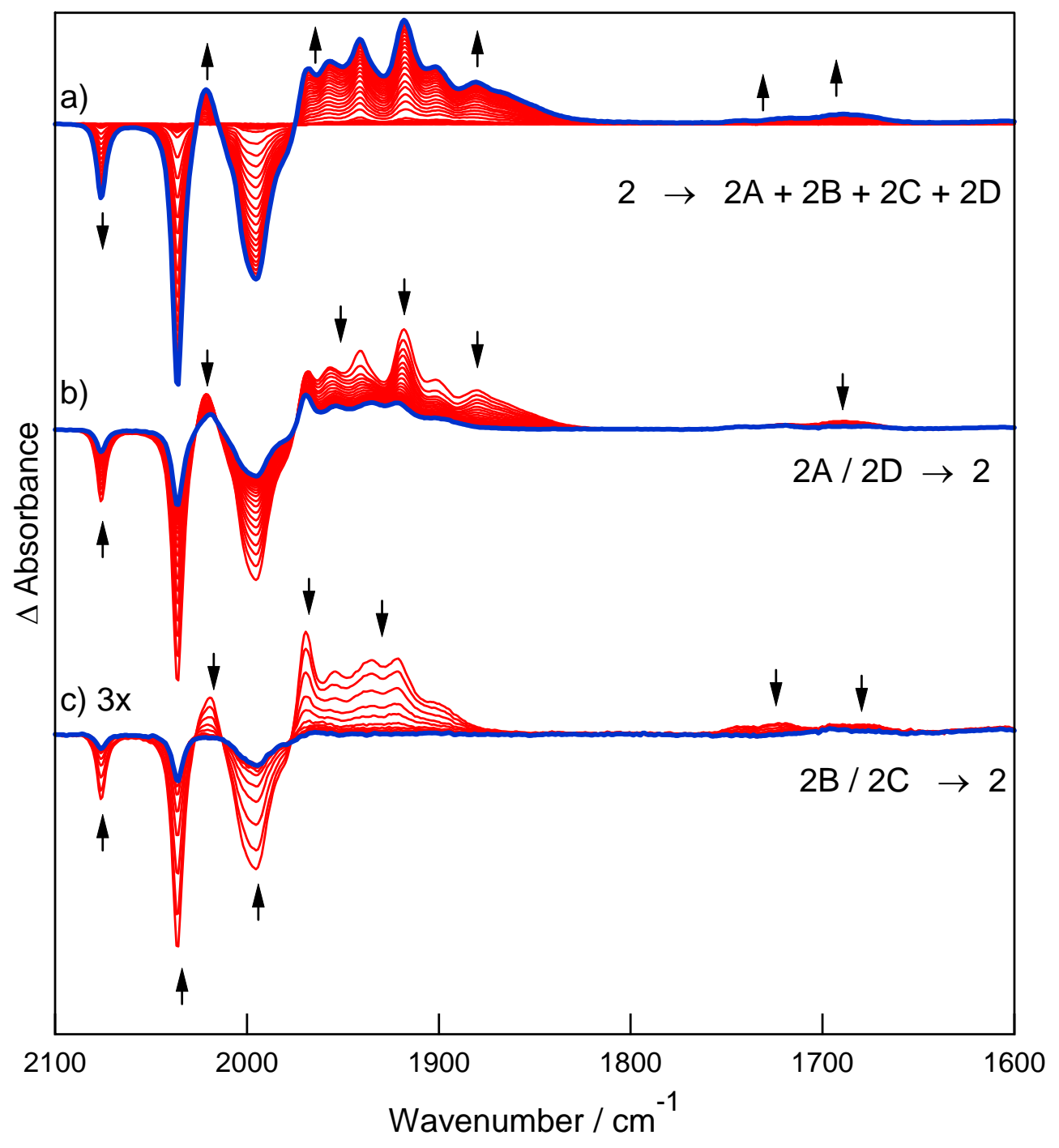

Fig. S1. Reduction of a $5 \mathrm{mM}$ solution of 2 in THF/0.2 $\mathrm{M} \mathrm{TBAClO}_{4}$ (40 p.s.i. Ar), Pt working, counter electrodes and Ag pseudo reference electrode. 


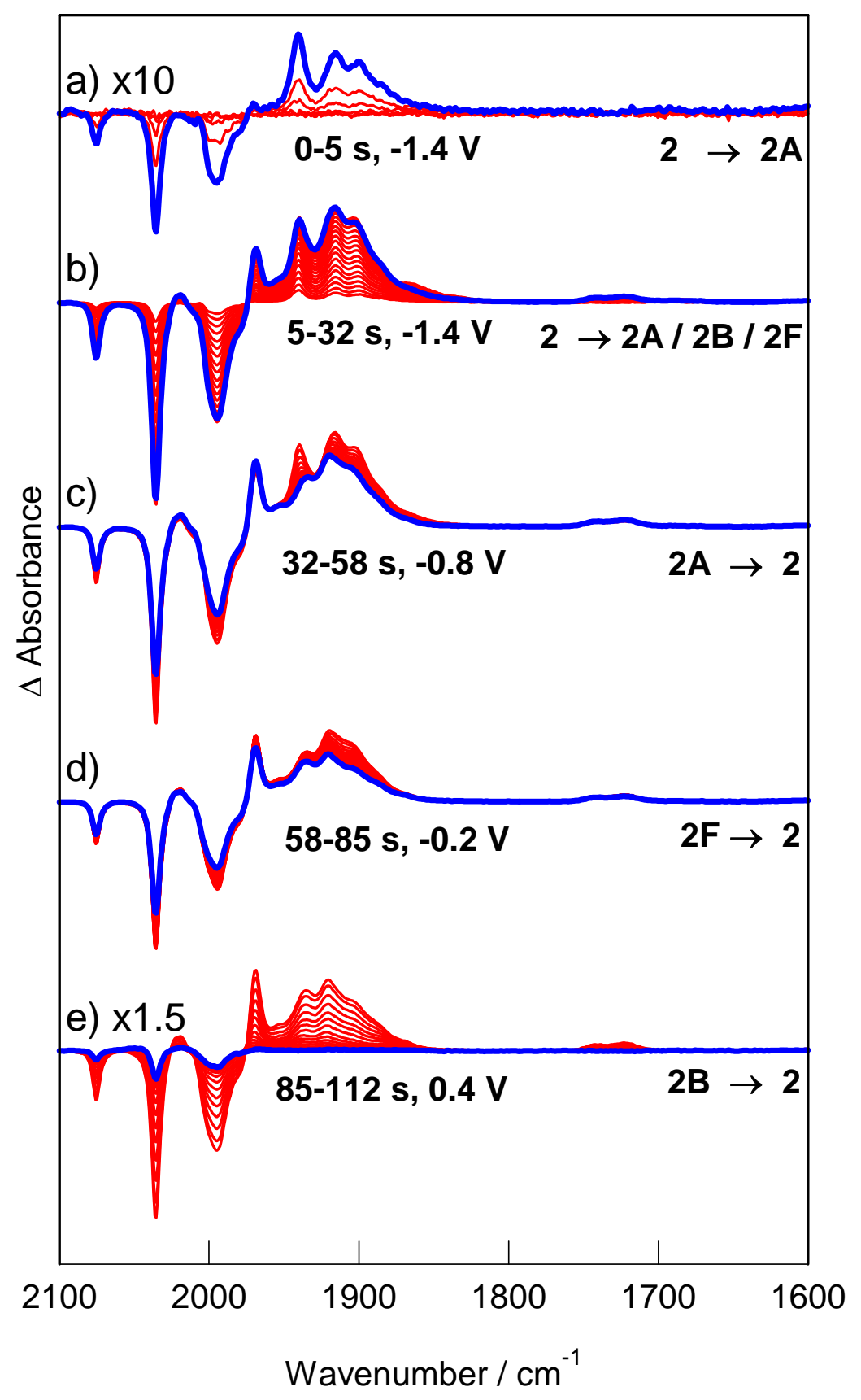

Fig. S2. Reduction of a $5 \mathrm{mM}$ solution of 2 in THF/0.2 $\mathrm{M} \mathrm{TBAClO}_{4}$ (40 p.s.i. CO), Pt working, counter electrodes and Ag pseudo reference electrode. 


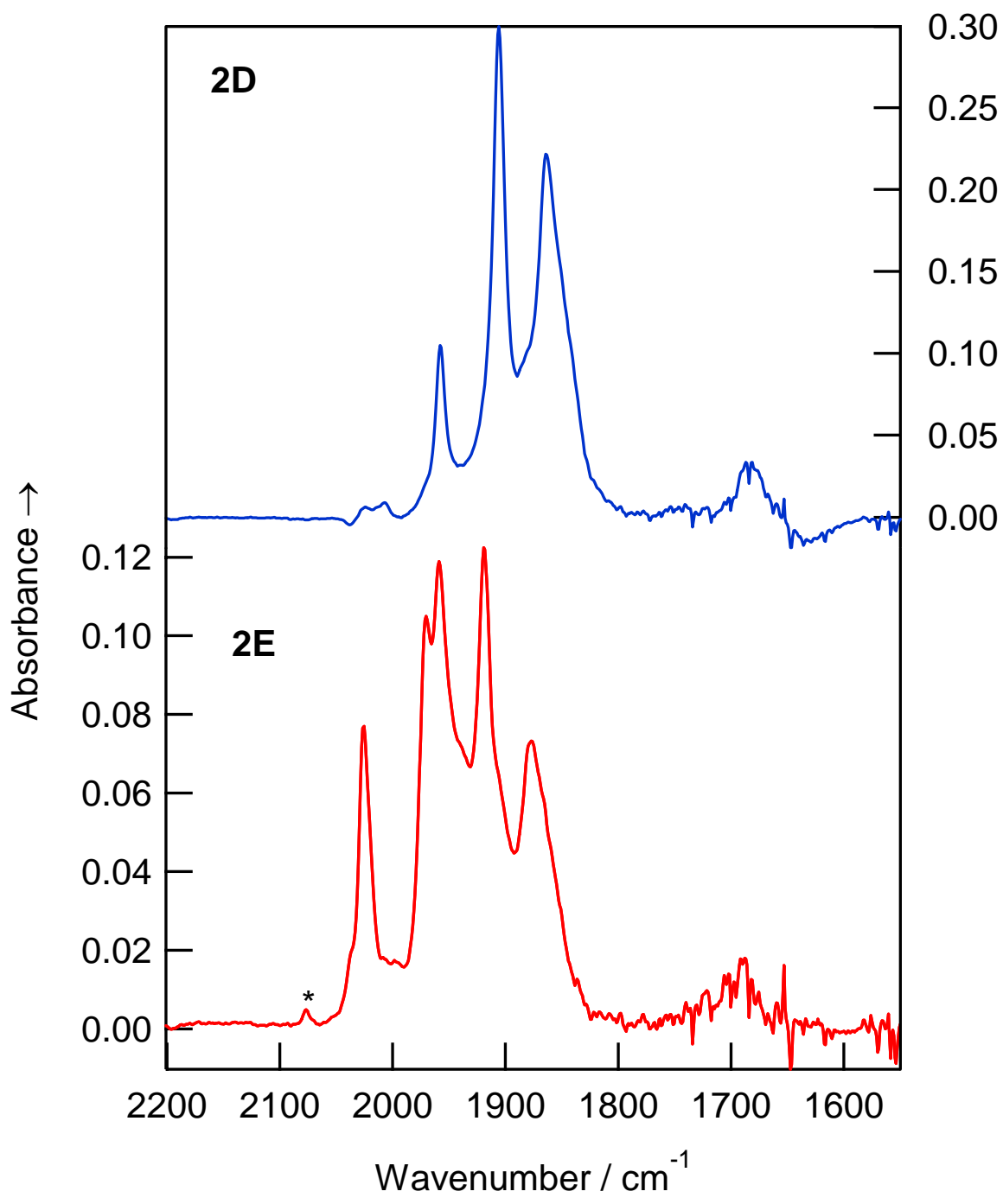

Fig. S3. Absorbance spectra obtained following reduction of a $5 \mathrm{mM}$ solution of 2 in $\mathrm{CH}_{3} \mathrm{CN} / 0.2 \mathrm{M}$ $\mathrm{TBAPF}_{6}$ in a continuous flow electrosynthesis experiment. The potential of the reticulated vitreous carbon working electrode was adjusted so as to optimise formation of (a) $2 \mathbf{D}$ or (b) 2E. Immediately following collection of the IR spectra the solution flow was directed to the EXAFS solution cell and the solution freeze quenched in isopentane $\left(-130^{\circ} \mathrm{C}\right)$, IR spectra were then collected by redirecting the flow from the electrosynthesis cell to the IR cell and these spectra were not significantly different from those formed immediately prior to EXAFS sample collection. Contamination by the neutral compound (into 2E) is indicated by the asterisk. The noise apparent in the spectrum near $1700 \mathrm{~cm}^{-1}$ is due to the low transmission at wavenumbers corresponding to the strong bands due to atmospheric water vapour. These present difficulties in these experiments owing to the need to operate the IR spectrometer with the sample compartment open. 

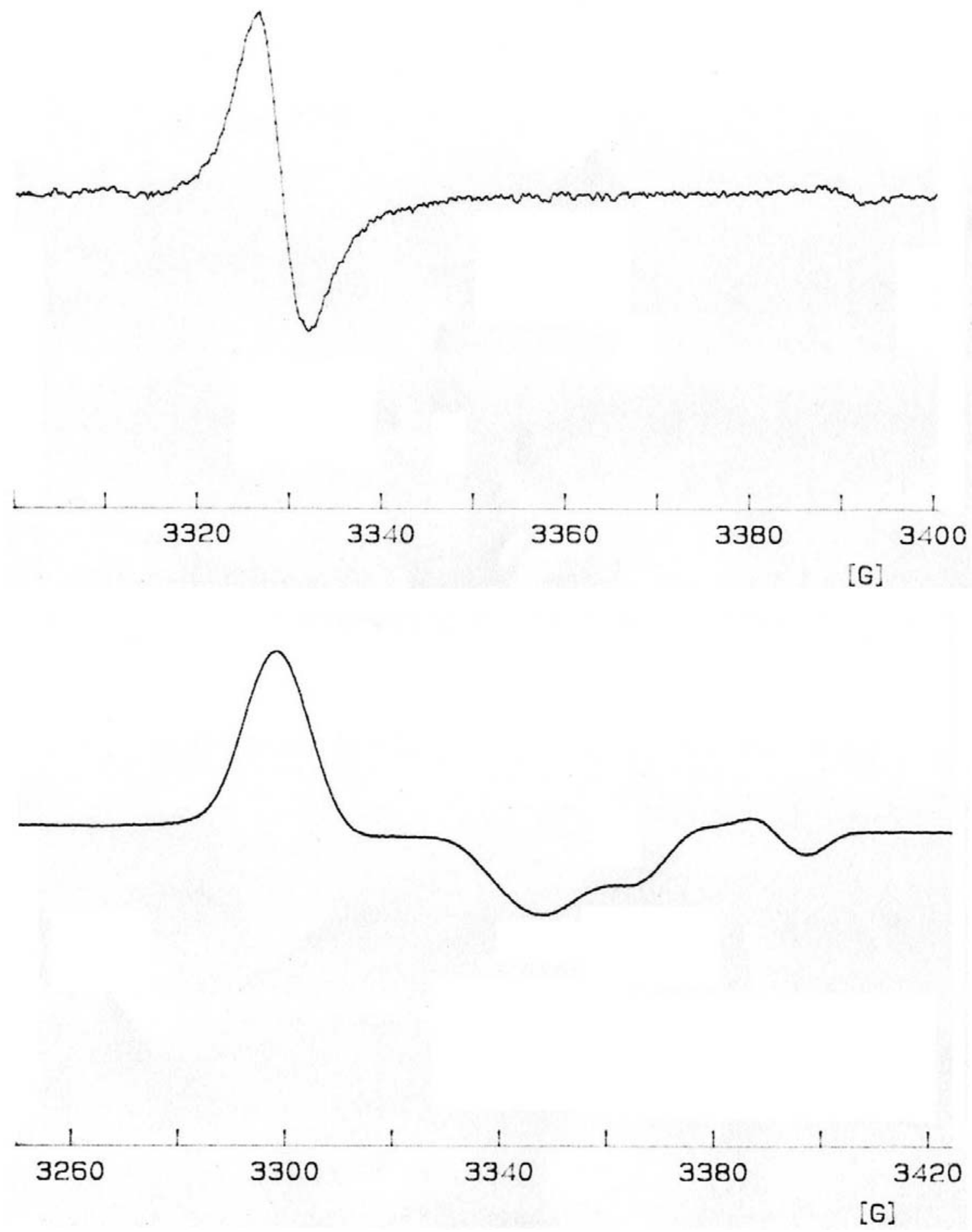

Fig. S4. EPR spectrum of $2 \mathbf{E}$ in $\mathrm{CH}_{3} \mathrm{CN}$ at room temperature (top) and at $120 \mathrm{~K}$ (bottom). 
Table S1. Description of the models, refinement statistics and final parameters for the EXAFS analysis of $2 D$.

2D-A

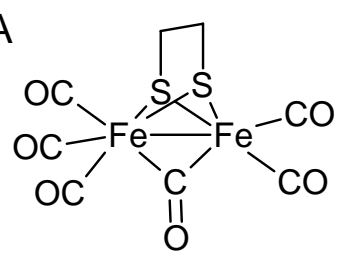

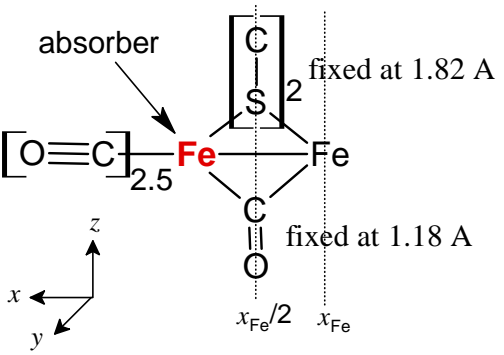

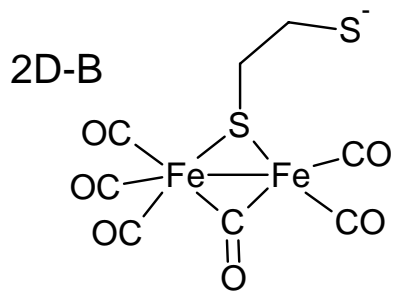

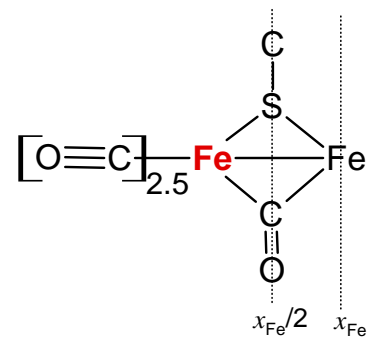

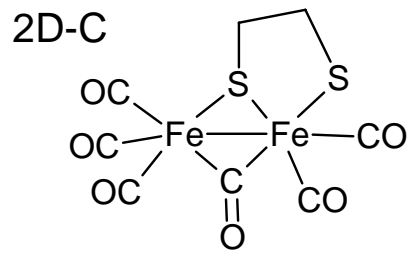

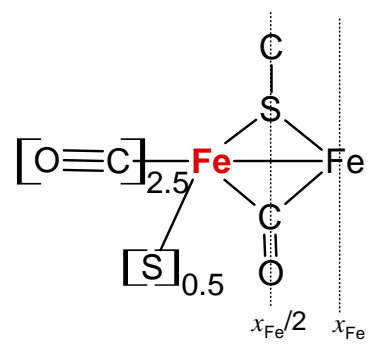

Definition of models used for EXAFS analysis of 2D. Unless otherwise specified the populations of the scattering atoms were set at 1 .

\begin{tabular}{|c|c|c|c|}
\hline Data & 2D-A & 2D-B & 2D-C \\
\hline$E_{0} / \mathrm{eV}$ & -9.33 & -11.60 & -11.02 \\
\hline$S_{0}^{2}$ & 0.83 & 0.90 & 0.87 \\
\hline$\chi^{2}\left[R_{X A F S}\right](\%)$ & $0.93[9.77]$ & 1.16 [10.94] & 1.01 [10.19] \\
\hline $\mathrm{Fe}-\mathrm{Fe} / \AA \AA A,(D W)$ & $2.57(0.0016)$ & $2.56(0.0010)$ & $2.56(0.0017)$ \\
\hline Fe- $\mu-S / A ̊,(D W)$ & $2.28(0.0054)$ & $2.26(0.0015)$ & $2.24(0.0010)$ \\
\hline Fe-S/Å, (DW) & & & $2.37(0.0010)$ \\
\hline $\mathrm{Fe}-\mathrm{C}(\mathrm{O}) / \mathrm{A},(\mathrm{DW})$ & $1.76(0.0014)$ & $1.75(0.0014)$ & $1.75(0.0015)$ \\
\hline $\mathrm{Fe}-\mathrm{O}(\mathrm{C}) / \mathrm{A},(\mathrm{DW})$ & $2.93(0.0028)$ & $2.91(0.0025)$ & $2.91(0.0026)$ \\
\hline $\mathrm{Fe}-\mathrm{C}_{\mathrm{br}} / \mathrm{A},(\mathrm{DW})$ & $2.39\left(0.001^{\text {fixed }}\right)$ & $2.37\left(0.001^{\text {fixed }}\right)$ & $2.39\left(0.001^{\text {fixed }}\right)$ \\
\hline $\mathbf{n}_{\text {refined }}$ & 11 & 11 & 13 \\
\hline
\end{tabular}

In each case the data were refined for the range 0.5 to $12.5 \AA^{-1}$ in $\mathrm{k}$ and 0.5 to $4 \AA$ in $\mathrm{R}$. The parameters refined for 2D-A were: $\mathrm{E}_{0}, \mathrm{~S}_{0}{ }^{2}, \mathrm{C}(\mathrm{x}), \sigma^{2}(\mathrm{C}), \mathrm{O}(\mathrm{x}), \sigma^{2}(\mathrm{O}), \mathrm{S}_{\mathrm{br}}(\mathrm{z}), \sigma^{2}\left(\mathrm{~S}_{\mathrm{br}}\right), \mathrm{Fe}(\mathrm{x}) \sigma^{2}(\mathrm{Fe}), \mathrm{C}_{\mathrm{br}}(\mathrm{z})$. The values of the populations and remaining parameters were fixed by constraints according to the descriptions of the models given above. For models $2 \mathbf{D}-\mathbf{B}$ and $2 \mathbf{D}-\mathbf{C}$ the additional parameters $\mathrm{S}_{\mathrm{t}}(\mathrm{y})$, $\sigma^{2}\left(S_{t}\right)$ were required. 
Table S2. Description of the model, refinement statistics and final parameters for the EXAFS analysis of $\mathbf{2 E}$.

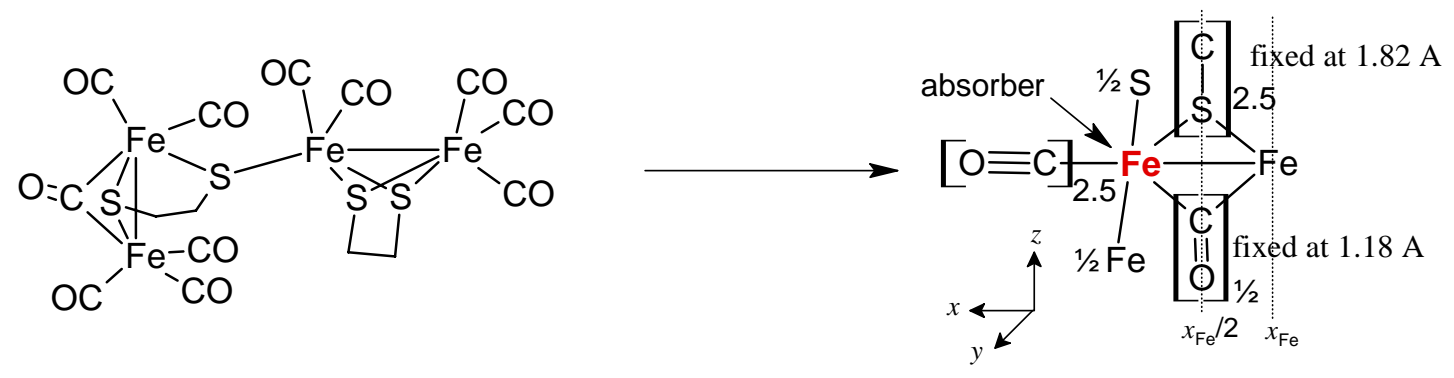

Model used for EXAFS analysis of 2E. Unless specified the populations of the scattering atoms equal 1.

\begin{tabular}{|c|c|c|c|c|c|c|c|}
\hline Initial Fe (long) & $2.8 \AA$ & $3.0 \AA$ & $3.2 \AA$ & $3.4 \AA$ & $3.6 \AA$ & $3.8 \AA$ & $4.0 \AA$ \\
\hline$E_{0} / \mathrm{eV}$ & -8.97 & -9.00 & -8.44 & -8.37 & -8.81 & -8.82 & -9.23 \\
\hline$S_{0}{ }^{2}$ & 0.91 & 0.91 & 0.90 & 0.90 & 0.91 & 0.91 & 0.91 \\
\hline$\chi^{2}$ & 1.00 & 0.93 & 0.69 & 0.71 & 1.09 & 1.14 & 1.38 \\
\hline$\left[R_{X A F S}\right](\%)$ & [8.77] & [8.47] & [7.33] & [7.42] & [9.15] & [9.35] & [10.30] \\
\hline Fe-Fe/Å & 2.53 & 2.53 & 2.52 & 2.52 & 2.51 & 2.51 & 2.52 \\
\hline (DW) & $(0.0025)$ & $(0.0025)$ & $(0.0038)$ & $(0.0038)$ & $(0.0027)$ & $(0.0027)$ & $(0.0026)$ \\
\hline $\mathbf{F e}-\mathrm{Fe}_{\text {long }} / \AA \mathbf{A}$ & 2.77 & 2.77 & 3.26 & 3.26 & 3.60 & 3.60 & 3.97 \\
\hline (DW) & $(0.001)$ & $(0.001)$ & $(0.0025)$ & $(0.0026)$ & $(0.0049)$ & $(0.0052)$ & $(0.0060)$ \\
\hline $\mathbf{F e}-\mu-\mathrm{S} / \AA \mathbf{A}$ & 2.24 & 2.24 & 2.24 & 2.22 & 2.24 & 2.24 & 2.25 \\
\hline (DW) & $(0.0027)$ & $(0.0028)$ & $(0.0021)$ & $(0.0028)$ & $(0.0022)$ & $(0.0022)$ & $(0.0031)$ \\
\hline Fe-S/Å & 2.36 & 2.36 & 2.37 & 2.37 & 2.39 & 2.40 & 2.39 \\
\hline (DW) & $(0.0119)$ & $(0.0116)$ & $(0.001)$ & $(0.001)$ & $(0.001)$ & $(0.001)$ & $(0.0153)$ \\
\hline $\mathrm{Fe}-\mathrm{C}(\mathbf{0}) / \AA$ & 1.77 & 1.77 & 1.77 & 1.77 & 1.77 & 1.77 & 1.77 \\
\hline (DW) & $(0.0024)$ & $(0.0025)$ & $(0.0024)$ & $(0.0023)$ & $(0.0024)$ & $(0.0024)$ & $(0.0023)$ \\
\hline $\mathrm{Fe}-\mathrm{O}(\mathrm{C}) / \AA \AA$ & 2.92 & 2.92 & 2.94 & 2.94 & 2.93 & 2.93 & 2.93 \\
\hline (DW) & $(0.0048)$ & $(0.0049)$ & $(0.0028)$ & $(0.0027)$ & $(0.0031)$ & $(0.0030)$ & $(0.0029)$ \\
\hline $\mathrm{Fe}-\mathrm{C}_{\mathrm{br}} / \AA$ & 2.14 & 2.14 & 2.17 & 2.17 & 2.10 & 2.10 & 2.12 \\
\hline (DW) & $\left(0.001^{\text {fixed }}\right)$ & $\left(0.001^{\text {fixed }}\right)$ & $\left(0.001^{\text {fixed }}\right)$ & $\left(0.001^{\text {fixed }}\right)$ & $\left(0.001^{\text {fixed }}\right)$ & $\left(0.001^{\text {fixed }}\right)$ & $\left(0.001^{\text {fixed }}\right)$ \\
\hline $\mathbf{n}_{\text {refined }}$ & 15 & 15 & 15 & 15 & 15 & 15 & 15 \\
\hline
\end{tabular}


Table S3. Computed Free Energies (Gas Phase, THF, and $\mathrm{CH}_{3} \mathrm{CN}$ Solution)

\begin{tabular}{|c|c|c|c|c|c|c|}
\hline & gas phase & gas phase & PCM & total in THF & PCM & total in $\mathrm{CH}_{3} \mathrm{CN}$ \\
\hline & $\Delta \mathbf{G}$ & $\Delta \mathbf{G}$ & $\Delta \mathbf{G}_{\text {solv }}(\mathrm{THF})$ & $\Delta \mathbf{G}$ & $\Delta \mathbf{G}_{\text {solv }}\left(\mathrm{CH}_{3} \mathrm{CN}\right)$ & $\Delta \mathbf{G}$ \\
\hline name & (au) & $\left(\mathrm{kcal} \mathrm{mol}^{-1}\right)$ & $\left(\mathrm{kcal} \mathrm{mol}^{-1}\right)$ & $\left(\mathrm{kcal} \mathrm{mol}^{-1}\right)$ & $\left(\mathrm{kcal} \mathrm{mol}^{-1}\right)$ & $\left(\mathrm{kcal} \mathrm{mol}^{-1}\right)$ \\
\hline$[2(a)]$ & -1025.893 & -643748.0 & 8.4 & -643739.6 & 10.8 & -643737.2 \\
\hline$[2(\mathrm{a})]^{1-}$ & -1025.954 & -643786.3 & -22.0 & -643808.3 & -23.5 & -643809.8 \\
\hline$[2(a)]^{2-}$ & -1025.868 & -643732.3 & -117.1 & -643849.4 & -131.4 & -643863.7 \\
\hline$[2(b)]^{1-}$ & -1025.942 & -643778.4 & -23.2 & -643801.6 & -25.2 & -643803.7 \\
\hline$[2(b)]^{2-}$ & -1025.873 & -643735.6 & -121.5 & -643857.1 & -136.5 & -643872.1 \\
\hline$[2 \mathrm{H}(\mathrm{a})]^{1-}$ & -1026.526 & -644145.1 & -23.3 & -644168.4 & -24.7 & -644169.8 \\
\hline$[2 \mathrm{H}(\mathrm{b})]^{1-}$ & -1026.523 & -644143.2 & -25.6 & -644168.8 & -27.7 & -644170.9 \\
\hline$[2 \mathrm{H}(\mathrm{c})]^{1-}$ & -1026.526 & -644145.0 & -24.4 & -644169.4 & -26.5 & -644171.4 \\
\hline$[2 \mathrm{H}(\mathrm{d})]^{1-}$ & -1026.504 & -644131.0 & -24.6 & -644155.5 & -27.1 & -644158.0 \\
\hline$[2 \mathrm{H}(\mathrm{e})]^{1-}$ & -1026.507 & -644133.4 & -23.4 & -644156.8 & -25.4 & -644158.8 \\
\hline$[2 H(f)]^{1-}$ & -1026.506 & -644132.4 & -23.9 & -644156.3 & -26.2 & -644158.5 \\
\hline$[2 \mathrm{H}(\mathrm{g})]^{1-}$ & -1026.505 & -644132.0 & -30.2 & -644162.2 & -34.3 & -644166.3 \\
\hline$[2 \mathrm{H}(\mathrm{h})]^{1-}$ & -1139.861 & -715263.0 & -23.0 & -715286.0 & -25.6 & -715288.5 \\
\hline $\mathrm{CO}$ & -113.335 & -71118.0 & 2.1 & -71115.9 & 2.7 & -71115.3 \\
\hline
\end{tabular}


Table S4. Computed and Predicted v(CO) Stretching Frequencies

\begin{tabular}{|c|c|c|c|c|c|c|c|c|c|c|c|c|c|c|c|c|c|}
\hline & & \multicolumn{7}{|c|}{ computed } & & \multicolumn{8}{|c|}{ predicted } \\
\hline name & & $v(\mathrm{CO})$ & $v(\mathrm{CO})$ & $v(\mathrm{CO})$ & $v(\mathrm{CO})$ & $v(\mathrm{CO})$ & $v(\mathrm{CO})$ & $v(\mathrm{CO})$ & $v(\mathrm{H})$ & $v(\mathrm{CO})$ & $v(\mathrm{CO})$ & $v(\mathrm{CO})$ & $v(\mathrm{CO})$ & $v(\mathrm{CO})$ & $v(\mathrm{CO})$ & $v(\mathrm{CO})$ & $v(\mathrm{H})$ \\
\hline [2(a)] & $\mathrm{cm}^{-1}$ & 2149 & 2104 & 2096 & 2087 & 2079 & 2076 & & & 2072 & 2029 & 2021 & 2012 & 2005 & 2001 & & \\
\hline & intensity & 492 & 2213 & 1174 & 846 & 0 & 34 & & & 492 & 2213 & 1174 & 846 & 0 & 34 & & \\
\hline$[2(a)]^{1-}$ & $\mathrm{cm}^{-1}$ & 2074 & 2014 & 1996 & 1994 & 1990 & 1982 & & & 2000 & 1942 & 1924 & 1922 & 1919 & 1911 & & \\
\hline & intensity & 678 & 1625 & 31 & 1800 & 1110 & 1793 & & & 678 & 1625 & 31 & 1800 & 1110 & 1793 & & \\
\hline$[2(a)]^{2-}$ & $\mathrm{cm}^{-1}$ & 1997 & 1973 & 1926 & 1921 & 1895 & 1888 & & & 1926 & 1902 & 1857 & 1852 & 1827 & 1820 & & \\
\hline & intensity & 582 & 1582 & 459 & 1483 & 1217 & 857 & & & 582 & 1582 & 459 & 1483 & 1217 & 857 & & \\
\hline$[2(b)]^{1-}$ & $\mathrm{cm}^{-1}$ & 2083 & 2045 & 2022 & 2020 & 2010 & 1880 & & & 2008 & 1971 & 1949 & 1947 & 1938 & 1813 & & \\
\hline & intensity & 725 & 1816 & 690 & 1446 & 167 & 626 & & & 725 & 1816 & 690 & 1446 & 167 & 626 & & \\
\hline$[2(b)]^{2-}$ & $\mathrm{cm}^{-1}$ & 2016 & 1964 & 1942 & 1934 & 1922 & 1779 & & & 1944 & 1894 & 1873 & 1865 & 1853 & 1715 & & \\
\hline & intensity & 656 & 2372 & 1279 & 830 & 845 & 499 & & & 656 & 2372 & 1279 & 830 & 845 & 499 & & \\
\hline$[2 \mathrm{H}(\mathrm{a})]^{1-}$ & $\mathrm{cm}^{-1}$ & 2075 & 2026 & 2019 & 2000 & 2000 & 1703 & & & 2000 & 1953 & 1946 & 1928 & 1928 & 1641 & & \\
\hline & intensity & 960 & 1853 & 1504 & 464 & 1 & 366 & & & 960 & 1853 & 1504 & 464 & 1 & 366 & & \\
\hline$[2 \mathrm{H}(\mathrm{b})]^{1-}$ & $\mathrm{cm}^{-1}$ & 2084 & 2044 & 2022 & 2012 & 2003 & 1705 & & & 2009 & 1971 & 1949 & 1939 & 1931 & 1644 & & \\
\hline & intensity & 644 & 1892 & 830 & 750 & 589 & 425 & & & 644 & 1892 & 830 & 750 & 589 & 425 & & \\
\hline$[2 \mathrm{H}(\mathrm{c})]^{1-}$ & $\mathrm{cm}^{-1}$ & 2096 & 2062 & 2043 & 2025 & 2022 & 1884 & & & 2021 & 1988 & 1970 & 1953 & 1949 & 1816 & & \\
\hline & intensity & 481 & 1984 & 966 & 618 & 767 & 507 & & & 481 & 1984 & 966 & 618 & 767 & 507 & & \\
\hline$[2 \mathrm{H}(\mathrm{d})]^{1-}$ & $\mathrm{cm}^{-1}$ & 2108 & 2060 & 2053 & 2024 & 1963 & 1939 & & 1928 & 2033 & 1986 & 1979 & 1952 & 1892 & 1869 & & 1859 \\
\hline & intensity & 808 & 795 & 848 & 1089 & 723 & 1158 & & 86 & 808 & 795 & 848 & 1089 & 723 & 1158 & & 86 \\
\hline$[2 \mathrm{H}(\mathrm{e})]^{1-}$ & $\mathrm{cm}^{-1}$ & 2108 & 2068 & 2054 & 2021 & 1964 & 1946 & & 1863 & 2032 & 1994 & 1980 & 1949 & 1894 & 1876 & & 1797 \\
\hline & intensity & 861 & 754 & 804 & 1143 & 836 & 978 & & 88 & 861 & 754 & 804 & 1143 & 836 & 978 & & 88 \\
\hline$[2 \mathrm{H}(\mathrm{f})]^{1-}$ & $\mathrm{cm}^{-1}$ & 2106 & 2062 & 2046 & 2021 & 1965 & 1946 & & 1914 & 2030 & 1988 & 1973 & 1948 & 1894 & 1876 & & 1845 \\
\hline & intensity & 578 & 816 & 1051 & 1040 & 843 & 958 & & 105 & 578 & 816 & 1051 & 1040 & 843 & 958 & & 105 \\
\hline$[2 \mathrm{H}(\mathrm{g})]^{1-}$ & $\mathrm{cm}^{-1}$ & 2060 & 2011 & 1995 & 1975 & 1968 & 1833 & & & 1986 & 1938 & 1923 & 1904 & 1897 & 1767 & & \\
\hline & \begin{tabular}{|l} 
intensity \\
\end{tabular} & 663 & 2253 & 965 & 524 & 1002 & 545 & & & 663 & 2253 & 965 & 524 & 1002 & 545 & & \\
\hline$[2 \mathrm{H}(\mathrm{h})]^{1-}$ & $\mathrm{cm}^{-1}$ & 2087 & 2037 & 2018 & 2009 & 2005 & 1984 & 1840 & & 2012 & 1964 & 1945 & 1937 & 1933 & 1913 & 1774 & \\
\hline & intensity & 379 & 2212 & 1275 & 1381 & 472 & 46 & 598 & & 379 & 2212 & 1275 & 1381 & 472 & 46 & 598 & \\
\hline axial,basal & $\mathrm{cm}^{-1}$ & 1826 & 1985 & 1987 & 1992 & 1999 & 2006 & & & 1760 & 1914 & 1916 & 1921 & 1927 & 1934 & & \\
\hline$[2 \mathrm{~B}+2]^{2-}$ & intensity & 512 & 52 & 12 & 507 & 1826 & 1287 & & & 512 & 52 & 12 & 507 & 1826 & 1287 & & \\
\hline & $\mathrm{cm}^{-1}$ & 2009 & 2018 & 2026 & 2044 & 2075 & 2080 & & & 1937 & 1945 & 1953 & 1971 & 2001 & 2005 & & \\
\hline & intensity & 524 & 1476 & 2175 & 1584 & 788 & 315 & & & 524 & 1476 & 2175 & 1584 & 788 & 315 & & \\
\hline axial,basal & $\mathrm{cm}^{-1}$ & 2004 & 2017 & 2032 & 2036 & 2042 & 2054 & & & 1932 & 1945 & 1959 & 1963 & 1969 & 1980 & & \\
\hline$[2 \mathrm{~B}+2]^{1-}$ & intensity & 506 & 472 & 1175 & 311 & 415 & 911 & & & 506 & 472 & 1175 & 311 & 415 & 911 & & \\
\hline & $\mathrm{cm}^{-1}$ & 2062 & 2074 & 2077 & 2091 & 2097 & 2136 & & & 1988 & 1999 & 2002 & 2016 & 2022 & 2059 & & \\
\hline & intensity & 1118 & 1161 & 574 & 991 & 1691 & 512 & & & & & & & & & & \\
\hline apical & $\mathrm{cm}^{-1}$ & 1801 & 1947 & 1957 & 1967 & 1987 & 1997 & & & 1736 & 1877 & 1886 & 1896 & 1916 & 1925 & & \\
\hline$[2 \mathrm{D}+2]^{2-}$ & intensity & 630 & 1033 & 449 & 1011 & 1935 & 409 & & & 630 & 1033 & 449 & 1011 & 1935 & 409 & & \\
\hline & $\mathrm{cm}^{-1}$ & 2005 & 2023 & 2033 & 2042 & 2084 & & & & 1933 & 1950 & 1960 & 1968 & 2009 & & & \\
\hline & intensity & 427 & 1385 & 680 & 1735 & 1260 & & & & 427 & 1385 & 680 & 1735 & 1260 & & & \\
\hline apical & $\mathrm{cm}^{-1}$ & 1919 & 2023 & 2028 & 2031 & 2035 & 2041 & & & 1850 & 1950 & 1956 & 1958 & 1962 & 1968 & & \\
\hline$[2 \mathrm{D}+2]^{1-}$ & intensity & 786 & 56 & 362 & 328 & 168 & 299 & & & 786 & 56 & 362 & 328 & 168 & 299 & & \\
\hline & $\mathrm{cm}^{-1}$ & 2045 & 2048 & 2065 & 2091 & 2107 & & & & 1972 & 1974 & 1991 & 2016 & 2031 & & & \\
\hline & intensity & 2886 & 1337 & 921 & 2134 & 585 & & & & 2886 & 1337 & 921 & 2134 & 585 & & & \\
\hline
\end{tabular}


Table S5. DFT optimized structures (in PDB format)

\begin{tabular}{|c|c|c|c|c|c|c|c|}
\hline \multicolumn{8}{|l|}{ HEADER } \\
\hline HETATM & 1 & $\mathrm{H}$ & 2 & 2 & -1.169 & 0.887 & 3.266 \\
\hline HETATM & 2 & $\mathrm{H}$ & 2 & 2 & -1.169 & $-\odot .887$ & 3.266 \\
\hline HETATM & 3 & $\mathrm{H}$ & 2 & 2 & 1.169 & 0.887 & 3.266 \\
\hline HETATM & 4 & $\mathrm{H}$ & 2 & 2 & 1.169 & -0.887 & 3.266 \\
\hline HETATM & 5 & C & 2 & 2 & $-\odot .772$ & $\odot . \odot \odot \odot$ & 2.760 \\
\hline HETATM & 6 & C & 2 & 2 & 0.772 & $\odot .00 \odot$ & 2.760 \\
\hline HETATM & 7 & FE & 2 & 2 & $\odot .00 \odot$ & 1.246 & -0.178 \\
\hline HETATM & 8 & FE & 2 & 2 & $\odot . \odot \odot \odot$ & -1.246 & -0.178 \\
\hline HETATM & 9 & $\mathrm{~S}$ & 2 & 2 & -1.504 & $\odot . \odot \odot \odot$ & 1.050 \\
\hline HETATM & 10 & $\mathrm{~s}$ & 2 & 2 & 1.504 & $\odot .0 \odot \odot$ & 1.050 \\
\hline HETATM & 11 & 0 & 2 & 2 & $\odot . \odot \odot \odot$ & 3.773 & 1.320 \\
\hline HETATM & 12 & C & 2 & 2 & $\odot . \odot \odot \odot$ & 2.788 & $\odot .732$ \\
\hline HETATM & 13 & 0 & 2 & 2 & -2.127 & 1.853 & -2.117 \\
\hline HETATM & 14 & C & 2 & 2 & -1.296 & 1.613 & -1.363 \\
\hline HETATM & 15 & 0 & 2 & 2 & 2.127 & 1.853 & -2.117 \\
\hline HETATM & 16 & C & 2 & 2 & 1.296 & 1.613 & -1.363 \\
\hline HETATM & 17 & 0 & 2 & 2 & -2.127 & -1.853 & -2.117 \\
\hline HETATM & 18 & C & 2 & 2 & -1.296 & -1.613 & -1.363 \\
\hline HETATM & 19 & 0 & 2 & 2 & 2.127 & -1.853 & -2.117 \\
\hline HETATM & 20 & C & 2 & 2 & 1.296 & -1.613 & -1.363 \\
\hline HETATM & 21 & 0 & 2 & 2 & $\odot . \odot \odot \odot$ & -3.773 & 1.320 \\
\hline HETATM & 22 & C & 2 & 2 & $\odot . \odot \odot \odot$ & -2.788 & $\odot .732$ \\
\hline
\end{tabular}




$$
\text { [2(a)]1- }
$$

$\begin{array}{lrrrrrrr}\text { HEADER } & & & & & & \\ \text { HETATM } & 1 & \mathrm{C} & 2 & 2 & -0.084 & 2.684 & 0.771 \\ \text { HETATM } & 2 & \mathrm{C} & 2 & 2 & 0.084 & 2.684 & -0.771 \\ \text { HETATM } & 3 & \mathrm{FE} & 2 & 2 & -1.386 & -0.171 & -0.068 \\ \text { HETATM } & 4 & \mathrm{FE} & 2 & 2 & 1.386 & -0.171 & 0.068 \\ \text { HETATM } & 5 & \mathrm{~S} & 2 & 2 & -0.086 & 0.980 & 1.517 \\ \text { HETATM } & 6 & \mathrm{~S} & 2 & 2 & 0.086 & 0.980 & -1.517 \\ \text { HETATM } & 7 & 0 & 2 & 2 & -3.910 & 1.381 & -0.035 \\ \text { HETATM } & 8 & \mathrm{C} & 2 & 2 & -2.881 & 0.845 & -0.066 \\ \text { HETATM } & 9 & 0 & 2 & 2 & -2.008 & -2.194 & 1.963 \\ \text { HETATM } & 10 & \mathrm{C} & 2 & 2 & -1.743 & -1.406 & 1.156 \\ \text { HETATM } & 11 & 0 & 2 & 2 & -2.033 & -2.011 & -2.256 \\ \text { HETATM } & 12 & \mathrm{C} & 2 & 2 & -1.763 & -1.294 & -1.385 \\ \text { HETATM } & 13 & 0 & 2 & 2 & 2.033 & -2.011 & 2.256 \\ \text { HETATM } & 14 & \mathrm{C} & 2 & 2 & 1.763 & -1.294 & 1.385 \\ \text { HETATM } & 15 & 0 & 2 & 2 & 2.007 & -2.194 & -1.963 \\ \text { HETATM } & 16 & \mathrm{C} & 2 & 2 & 1.743 & -1.405 & -1.156 \\ \text { HETATM } & 17 & 0 & 2 & 2 & 3.910 & 1.381 & 0.034 \\ \text { HETATM } & 18 & \mathrm{C} & 2 & 2 & 2.881 & 0.845 & 0.066\end{array}$

[2(a) ]2-

$\begin{array}{lrrrrrrr}\text { HEADER } & & & & & & \\ \text { HETATM } & 1 & \mathrm{H} & 2 & 2 & 1.172 & 0.895 & -2.894 \\ \text { HETATM } & 2 & \mathrm{H} & 2 & 2 & 1.154 & -0.897 & -2.924 \\ \text { HETATM } & 3 & \mathrm{H} & 2 & 2 & -1.154 & 0.897 & -2.924 \\ \text { HETATM } & 4 & \mathrm{H} & 2 & 2 & -1.172 & -0.895 & -2.894 \\ \text { HETATM } & 5 & \mathrm{C} & 2 & 2 & 0.782 & -0.005 & -2.398 \\ \text { HETATM } & 6 & \mathrm{C} & 2 & 2 & -0.782 & 0.005 & -2.398 \\ \text { HETATM } & 7 & \mathrm{FE} & 2 & 2 & 0.000 & 1.741 & 0.155 \\ \text { HETATM } & 8 & \mathrm{FE} & 2 & 2 & 0.000 & -1.741 & 0.155 \\ \text { HETATM } & 9 & \mathrm{~S} & 2 & 2 & 1.480 & -0.029 & -0.673 \\ \text { HETATM } & 10 & \mathrm{~S} & 2 & 2 & -1.480 & 0.029 & -0.673 \\ \text { HETATM } & 11 & 0 & 2 & 2 & -1.202 & 3.831 & -1.506 \\ \text { HETATM } & 12 & \mathrm{C} & 2 & 2 & -0.673 & 2.958 & -0.915 \\ \text { HETATM } & 13 & 0 & 2 & 2 & 2.267 & 3.410 & 0.927 \\ \text { HETATM } & 14 & \mathrm{C} & 2 & 2 & 1.349 & 2.750 & 0.623 \\ \text { HETATM } & 15 & 0 & 2 & 2 & -2.267 & -3.410 & 0.927 \\ \text { HETATM } & 16 & \mathrm{C} & 2 & 2 & -1.349 & -2.750 & 0.623 \\ \text { HETATM } & 17 & 0 & 2 & 2 & 1.202 & -3.831 & -1.506 \\ \text { HETATM } & 18 & \mathrm{C} & 2 & 2 & 0.673 & -2.958 & -0.915 \\ \text { HETATM } & 19 & \mathrm{C} & 2 & 2 & -0.676 & 1.650 & 1.779 \\ \text { HETATM } & 20 & \mathrm{C} & 2 & 2 & 0.676 & -1.650 & 1.779 \\ \text { HETATM } & 21 & 0 & 2 & 2 & -1.190 & 1.743 & 2.829 \\ \text { HETATM } & 22 & 0 & 2 & 2 & 1.190 & -1.743 & 2.829\end{array}$


[2(b) ]1-

$\begin{array}{lrrrrrrr}\text { HEADER } & & & & & & \\ \text { HETATM } & 1 & \mathrm{H} & 2 & 2 & -3.013 & -3.067 & 0.597 \\ \text { HETATM } & 2 & \mathrm{H} & 2 & 2 & -3.143 & -2.076 & -0.876 \\ \text { HETATM } & 3 & \mathrm{H} & 2 & 2 & -0.536 & -2.955 & 0.507 \\ \text { HETATM } & 4 & \mathrm{H} & 2 & 2 & -1.125 & -3.520 & -1.081 \\ \text { HETATM } & 5 & \mathrm{C} & 2 & 2 & -2.537 & -2.257 & 0.023 \\ \text { HETATM } & 6 & \mathrm{C} & 2 & 2 & -1.112 & -2.672 & -0.378 \\ \text { HETATM } & 7 & \mathrm{FE} & 2 & 2 & 1.434 & -0.183 & 0.000 \\ \text { HETATM } & 8 & \mathrm{FE} & 2 & 2 & -0.983 & 0.572 & -0.027 \\ \text { HETATM } & 9 & \mathrm{~S} & 2 & 2 & -2.540 & -0.731 & 1.088 \\ \text { HETATM } & 10 & \mathrm{~S} & 2 & 2 & -0.274 & -1.261 & -1.264 \\ \text { HETATM } & 11 & 0 & 2 & 2 & 1.079 & -1.973 & 2.333 \\ \text { HETATM } & 12 & \mathrm{C} & 2 & 2 & 1.181 & -1.264 & 1.426 \\ \text { HETATM } & 13 & 0 & 2 & 2 & 3.216 & 1.548 & 1.534 \\ \text { HETATM } & 14 & \mathrm{C} & 2 & 2 & 2.505 & 0.866 & 0.928 \\ \text { HETATM } & 15 & 0 & 2 & 2 & 3.529 & -1.174 & -1.790 \\ \text { HETATM } & 16 & \mathrm{C} & 2 & 2 & 2.697 & -0.761 & -1.099 \\ \text { HETATM } & 17 & 0 & 2 & 2 & -2.743 & 1.794 & -2.064 \\ \text { HETATM } & 18 & \mathrm{C} & 2 & 2 & -1.994 & 1.359 & -1.299 \\ \text { HETATM } & 19 & 0 & 2 & 2 & 1.012 & 2.390 & -1.411 \\ \text { HETATM } & 20 & \mathrm{C} & 2 & 2 & 0.728 & 1.405 & -0.836 \\ \text { HETATM } & 21 & 0 & 2 & 2 & -1.211 & 2.692 & 1.979 \\ \text { HETATM } & 22 & \mathrm{C} & 2 & 2 & -1.125 & 1.853 & 1.188\end{array}$

[2(b) $] 2$ -

$\begin{array}{lrrrrrrr}\text { HEADER } & & & & & & & \\ \text { HETATM } & 1 & \mathrm{H} & 2 & 2 & -2.607 & 3.461 & -0.069 \\ \text { HETATM } & 2 & \mathrm{H} & 2 & 2 & -0.833 & 3.670 & -0.081 \\ \text { HETATM } & 3 & \mathrm{H} & 2 & 2 & -2.381 & 1.360 & -1.392 \\ \text { HETATM } & 4 & \mathrm{H} & 2 & 2 & -1.580 & 2.705 & -2.264 \\ \text { HETATM } & 5 & \mathrm{C} & 2 & 2 & -1.642 & 2.921 & -0.079 \\ \text { HETATM } & 6 & \mathrm{C} & 2 & 2 & -1.548 & 2.071 & -1.359 \\ \text { HETATM } & 7 & \mathrm{FE} & 2 & 2 & -0.169 & -1.190 & -0.863 \\ \text { HETATM } & 8 & \mathrm{FE} & 2 & 2 & 0.535 & 0.672 & 0.804 \\ \text { HETATM } & 9 & \mathrm{~S} & 2 & 2 & -1.533 & 1.874 & 1.445 \\ \text { HETATM } & 10 & \mathrm{~S} & 2 & 2 & 0.064 & 1.128 & -1.423 \\ \text { HETATM } & 11 & 0 & 2 & 2 & -3.114 & -1.207 & -0.980 \\ \text { HETATM } & 12 & \mathrm{C} & 2 & 2 & -1.949 & -1.151 & -0.903 \\ \text { HETATM } & 13 & 0 & 2 & 2 & -0.075 & -3.596 & 0.775 \\ \text { HETATM } & 14 & \mathrm{C} & 2 & 2 & -0.109 & -2.616 & 0.135 \\ \text { HETATM } & 15 & 0 & 2 & 2 & 0.771 & -2.370 & -3.368 \\ \text { HETATM } & 16 & \mathrm{C} & 2 & 2 & 0.438 & -1.855 & -2.368 \\ \text { HETATM } & 17 & 0 & 2 & 2 & 2.536 & 2.708 & 1.338 \\ \text { HETATM } & 18 & \mathrm{C} & 2 & 2 & 1.709 & 1.910 & 1.113 \\ \text { HETATM } & 19 & 0 & 2 & 2 & 2.687 & -1.175 & 0.110 \\ \text { HETATM } & 20 & \mathrm{C} & 2 & 2 & 1.592 & -0.695 & 0.086 \\ \text { HETATM } & 21 & 0 & 2 & 2 & 0.579 & -0.488 & 3.479 \\ \text { HETATM } & 22 & \mathrm{C} & 2 & 2 & 0.561 & -0.038 & 2.403\end{array}$




\begin{tabular}{|c|c|c|c|c|c|c|c|}
\hline \multicolumn{8}{|l|}{ HEADER } \\
\hline HETATM & 1 & $\mathrm{H}$ & 2 & 2 & -0.826 & 3.120 & 1.348 \\
\hline HETATM & 2 & $\mathrm{H}$ & 2 & 2 & 0.956 & 3.192 & 1.190 \\
\hline HETATM & 3 & H & 2 & 2 & -1.061 & 3.202 & -0.930 \\
\hline HETATM & 4 & $\mathrm{H}$ & 2 & 2 & 0.716 & 3.271 & -1.136 \\
\hline HETATM & 5 & C & 2 & 2 & 0.047 & 2.669 & $\odot .865$ \\
\hline HETATM & 6 & C & 2 & 2 & -0.112 & 2.722 & -0.669 \\
\hline HETATM & 7 & FE & 2 & 2 & -1.214 & -0.269 & 0.118 \\
\hline HETATM & 8 & FE & 2 & 2 & 1.338 & -0.183 & -0.147 \\
\hline HETATM & 9 & $S$ & 2 & 2 & 0.157 & 0.939 & 1.536 \\
\hline HETATM & 10 & $\mathrm{~S}$ & 2 & 2 & $-\odot .158$ & 1.044 & -1.466 \\
\hline HETATM & 11 & 0 & 2 & 2 & -2.954 & 1.946 & 0.377 \\
\hline HETATM & 12 & C & 2 & 2 & -2.850 & 0.731 & 0.325 \\
\hline HETATM & 13 & 0 & 2 & 2 & -1.895 & -2.134 & 2.260 \\
\hline HETATM & 14 & C & 2 & 2 & -1.612 & -1.397 & 1.413 \\
\hline HETATM & 15 & 0 & 2 & 2 & -2.339 & -1.986 & -1.959 \\
\hline HETATM & 16 & C & 2 & 2 & -1.881 & -1.307 & -1.140 \\
\hline HETATM & 17 & 0 & 2 & 2 & 2.057 & -2.223 & 1.826 \\
\hline HETATM & 18 & C & 2 & 2 & 1.756 & -1.424 & 1.043 \\
\hline HETATM & 19 & 0 & 2 & 2 & 1.615 & -2.077 & -2.362 \\
\hline HETATM & 20 & C & 2 & 2 & 1.489 & -1.336 & -1.480 \\
\hline HETATM & 21 & 0 & 2 & 2 & 3.861 & 1.300 & -0.361 \\
\hline HETATM & 22 & C & 2 & 2 & 2.839 & 0.759 & -0.272 \\
\hline HETATM & 23 & $\mathrm{H}$ & 2 & 2 & -3.818 & 0.135 & 0.407 \\
\hline
\end{tabular}

$[2 \mathrm{H}(\mathrm{b})] 1-$

$\begin{array}{lrrrrrrr}\text { HEADER } & & & & & & & \\ \text { HETATM } & 1 & \mathrm{H} & 2 & 2 & 3.510 & -1.274 & 0.463 \\ \text { HETATM } & 2 & \mathrm{H} & 2 & 2 & 3.056 & -1.268 & -1.257 \\ \text { HETATM } & 3 & \mathrm{H} & 2 & 2 & 3.283 & 1.057 & 0.647 \\ \text { HETATM } & 4 & \mathrm{H} & 2 & 2 & 3.412 & 1.045 & -1.127 \\ \text { HETATM } & 5 & \mathrm{C} & 2 & 2 & 2.794 & -0.873 & -0.268 \\ \text { HETATM } & 6 & \mathrm{C} & 2 & 2 & 2.829 & 0.672 & -0.275 \\ \text { HETATM } & 7 & \mathrm{FE} & 2 & 2 & -0.074 & 0.246 & 1.191 \\ \text { HETATM } & 8 & \mathrm{FE} & 2 & 2 & -0.191 & -0.250 & -1.287 \\ \text { HETATM } & 9 & \mathrm{~S} & 2 & 2 & 1.120 & -1.556 & 0.163 \\ \text { HETATM } & 10 & \mathrm{~S} & 2 & 2 & 1.136 & 1.440 & -0.369 \\ \text { HETATM } & 11 & 0 & 2 & 2 & 1.335 & 0.946 & 3.633 \\ \text { HETATM } & 12 & \mathrm{C} & 2 & 2 & 0.766 & 0.664 & 2.662 \\ \text { HETATM } & 13 & 0 & 2 & 2 & -2.029 & -1.539 & 2.441 \\ \text { HETATM } & 14 & \mathrm{C} & 2 & 2 & -1.263 & -0.839 & 1.935 \\ \text { HETATM } & 15 & 0 & 2 & 2 & -2.327 & 1.789 & 2.067 \\ \text { HETATM } & 16 & \mathrm{C} & 2 & 2 & -1.297 & 1.726 & 1.420 \\ \text { HETATM } & 17 & 0 & 2 & 2 & -2.302 & -2.267 & -1.088 \\ \text { HETATM } & 18 & \mathrm{C} & 2 & 2 & -1.473 & -1.462 & -1.148 \\ \text { HETATM } & 19 & 0 & 2 & 2 & -2.083 & 1.804 & -2.169 \\ \text { HETATM } & 20 & \mathrm{C} & 2 & 2 & -1.341 & 0.994 & -1.808 \\ \text { HETATM } & 21 & 0 & 2 & 2 & 1.022 & -0.937 & -3.870 \\ \text { HETATM } & 22 & \mathrm{C} & 2 & 2 & 0.581 & -0.654 & -2.837 \\ \text { HETATM } & 23 & \mathrm{H} & 2 & 2 & -0.982 & 2.664 & 0.853\end{array}$




\begin{tabular}{|c|c|c|c|c|c|c|c|}
\hline \multicolumn{8}{|l|}{ HEADER } \\
\hline HETATM & 1 & $\mathrm{H}$ & 2 & 2 & -1.479 & 2.589 & -0.066 \\
\hline HETATM & 2 & $\mathrm{H}$ & 2 & 2 & -0.652 & 3.458 & -1.395 \\
\hline HETATM & 3 & $\mathrm{H}$ & 2 & 2 & 0.176 & 4.202 & 0.885 \\
\hline HETATM & 4 & $\mathrm{H}$ & 2 & 2 & 1.476 & 3.444 & -0.065 \\
\hline HETATM & 5 & C & 2 & 2 & -0.519 & 2.747 & -0.565 \\
\hline HETATM & 6 & C & 2 & 2 & 0.521 & 3.252 & 0.446 \\
\hline HETATM & 7 & FE & 2 & 2 & -1.249 & -0.671 & -0.507 \\
\hline HETATM & 8 & FE & 2 & 2 & 1.022 & 0.049 & 0.484 \\
\hline HETATM & 9 & S & 2 & 2 & $\odot .042$ & 1.141 & -1.328 \\
\hline HETATM & 10 & $\mathrm{~S}$ & 2 & 2 & $\odot .762$ & 2.049 & 1.835 \\
\hline HETATM & 11 & 0 & 2 & 2 & -3.737 & $\odot .794$ & $\odot .088$ \\
\hline HETATM & 12 & C & 2 & 2 & -2.727 & $\odot .271$ & -0.118 \\
\hline HETATM & 13 & 0 & 2 & 2 & -1.990 & -3.120 & 0.901 \\
\hline HETATM & 14 & C & 2 & 2 & -1.698 & -2.161 & 0.324 \\
\hline HETATM & 15 & 0 & 2 & 2 & -1.804 & -1.677 & -3.208 \\
\hline HETATM & 16 & C & 2 & 2 & -1.567 & -1.291 & -2.145 \\
\hline HETATM & 17 & 0 & 2 & 2 & 3.772 & $\odot .797$ & -0.165 \\
\hline HETATM & 18 & C & 2 & 2 & 2.689 & $\odot .489$ & ๑. 087 \\
\hline HETATM & 19 & 0 & 2 & 2 & 1.250 & -2.428 & -1.088 \\
\hline HETATM & 20 & C & 2 & 2 & $\odot .816$ & -1.465 & -0.566 \\
\hline HETATM & 21 & 0 & 2 & 2 & 1.657 & -1.399 & 2.942 \\
\hline HETATM & 22 & C & 2 & 2 & 1.419 & $-\odot .835$ & 1.964 \\
\hline HETATM & 23 & $\mathrm{H}$ & 2 & 2 & -0.633 & -0.157 & $\odot .914$ \\
\hline
\end{tabular}

$[2 \mathrm{H}(\mathrm{d})] 1-$

$\begin{array}{lrrrrrrr}\text { HEADER } & & & & & & \\ \text { HETATM } & 1 & \mathrm{H} & 2 & 2 & 0.742 & 2.769 & -1.482 \\ \text { HETATM } & 2 & \mathrm{H} & 2 & 2 & -1.035 & 2.806 & -1.355 \\ \text { HETATM } & 3 & \mathrm{H} & 2 & 2 & 0.908 & 2.969 & 0.836 \\ \text { HETATM } & 4 & \mathrm{H} & 2 & 2 & -0.868 & 2.992 & 0.967 \\ \text { HETATM } & 5 & \mathrm{C} & 2 & 2 & -0.124 & 2.309 & -0.992 \\ \text { HETATM } & 6 & \mathrm{C} & 2 & 2 & -0.011 & 2.439 & 0.558 \\ \text { HETATM } & 7 & \mathrm{FE} & 2 & 2 & 1.716 & -0.162 & -0.088 \\ \text { HETATM } & 8 & \mathrm{FE} & 2 & 2 & -1.623 & -0.295 & 0.115 \\ \text { HETATM } & 9 & \mathrm{~S} & 2 & 2 & -0.143 & 0.535 & -1.554 \\ \text { HETATM } & 10 & \mathrm{~S} & 2 & 2 & 0.076 & 0.781 & 1.396 \\ \text { HETATM } & 11 & 0 & 2 & 2 & 3.839 & 1.740 & 0.590 \\ \text { HETATM } & 12 & \mathrm{C} & 2 & 2 & 2.942 & 1.062 & 0.264 \\ \text { HETATM } & 13 & 0 & 2 & 2 & 3.190 & -1.291 & -2.341 \\ \text { HETATM } & 14 & \mathrm{C} & 2 & 2 & 2.618 & -0.844 & -1.432 \\ \text { HETATM } & 15 & 0 & 2 & 2 & 2.064 & -2.577 & 1.527 \\ \text { HETATM } & 16 & \mathrm{C} & 2 & 2 & 1.849 & -1.649 & 0.851 \\ \text { HETATM } & 17 & 0 & 2 & 2 & -3.580 & 1.950 & 0.096 \\ \text { HETATM } & 18 & \mathrm{C} & 2 & 2 & -2.789 & 1.112 & 0.103 \\ \text { HETATM } & 19 & 0 & 2 & 2 & -2.884 & -2.008 & -1.885 \\ \text { HETATM } & 20 & \mathrm{C} & 2 & 2 & -2.421 & -1.312 & -1.093 \\ \text { HETATM } & 21 & 0 & 2 & 2 & -2.559 & -1.711 & 2.495 \\ \text { HETATM } & 22 & \mathrm{C} & 2 & 2 & -2.225 & -1.132 & 1.557 \\ \text { HETATM } & 23 & \mathrm{H} & 2 & 2 & -0.671 & -1.456 & 0.107\end{array}$




$$
[2 \mathrm{H}(\mathrm{e})] 1-
$$

$\begin{array}{lrrrrrrr}\text { HEADER } & & & & & & \\ \text { HETATM } & 1 & \mathrm{H} & 2 & 2 & 0.981 & -2.964 & 1.184 \\ \text { HETATM } & 2 & \mathrm{H} & 2 & 2 & -0.802 & -3.095 & 1.177 \\ \text { HETATM } & 3 & \mathrm{H} & 2 & 2 & 0.978 & -2.978 & -1.153 \\ \text { HETATM } & 4 & \mathrm{H} & 2 & 2 & -0.805 & -3.093 & -1.149 \\ \text { HETATM } & 5 & \mathrm{C} & 2 & 2 & 0.054 & -2.525 & 0.793 \\ \text { HETATM } & 6 & \mathrm{C} & 2 & 2 & 0.054 & -2.529 & -0.765 \\ \text { HETATM } & 7 & \mathrm{FE} & 2 & 2 & 1.605 & 0.159 & -0.028 \\ \text { HETATM } & 8 & \mathrm{FE} & 2 & 2 & -1.726 & -0.026 & 0.011 \\ \text { HETATM } & 9 & \mathrm{~S} & 2 & 2 & -0.041 & -0.804 & 1.495 \\ \text { HETATM } & 10 & \mathrm{~S} & 2 & 2 & -0.030 & -0.811 & -1.468 \\ \text { HETATM } & 11 & 0 & 2 & 2 & 3.893 & -1.566 & -0.653 \\ \text { HETATM } & 12 & \mathrm{C} & 2 & 2 & 2.921 & -0.988 & -0.361 \\ \text { HETATM } & 13 & 0 & 2 & 2 & 2.915 & 1.522 & 2.200 \\ \text { HETATM } & 14 & \mathrm{C} & 2 & 2 & 2.406 & 0.986 & 1.302 \\ \text { HETATM } & 15 & 0 & 2 & 2 & 1.922 & 2.473 & -1.782 \\ \text { HETATM } & 16 & \mathrm{C} & 2 & 2 & 1.735 & 1.578 & -1.054 \\ \text { HETATM } & 17 & 0 & 2 & 2 & -0.964 & 2.861 & 0.194 \\ \text { HETATM } & 18 & \mathrm{C} & 2 & 2 & -1.107 & 1.725 & 0.106 \\ \text { HETATM } & 19 & 0 & 2 & 2 & -3.596 & 0.250 & -2.221 \\ \text { HETATM } & 20 & \mathrm{C} & 2 & 2 & -2.861 & 0.186 & -1.336 \\ \text { HETATM } & 21 & 0 & 2 & 2 & -3.634 & 0.072 & 2.222 \\ \text { HETATM } & 22 & \mathrm{C} & 2 & 2 & -2.886 & 0.083 & 1.346 \\ \text { HETATM } & 23 & \mathrm{H} & 2 & 2 & -2.298 & -1.430 & -0.031\end{array}$

$\begin{array}{lrrrrrrr}\begin{array}{l}\text { 2H }(\mathrm{f}) \text { ] } 1- \\ \text { HEADER }\end{array} & & & & & & & \\ \text { HETATM } & 1 & \mathrm{H} & 2 & 2 & 1.080 & -2.951 & 0.773 \\ \text { HETATM } & 2 & \mathrm{H} & 2 & 2 & -0.665 & -3.284 & 0.628 \\ \text { HETATM } & 3 & \mathrm{H} & 2 & 2 & 1.155 & -2.629 & -1.531 \\ \text { HETATM } & 4 & \mathrm{H} & 2 & 2 & -0.615 & -2.784 & -1.653 \\ \text { HETATM } & 5 & \mathrm{C} & 2 & 2 & 0.126 & -2.553 & 0.409 \\ \text { HETATM } & 6 & \mathrm{C} & 2 & 2 & 0.201 & -2.273 & -1.124 \\ \text { HETATM } & 7 & \mathrm{FE} & 2 & 2 & 1.535 & 0.292 & 0.227 \\ \text { HETATM } & 8 & \mathrm{FE} & 2 & 2 & -1.746 & 0.051 & -0.059 \\ \text { HETATM } & 9 & \mathrm{~S} & 2 & 2 & -0.186 & -1.014 & 1.413 \\ \text { HETATM } & 10 & \mathrm{~S} & 2 & 2 & 0.100 & -0.459 & -1.524 \\ \text { HETATM } & 11 & 0 & 2 & 2 & 3.958 & -1.195 & -0.487 \\ \text { HETATM } & 12 & \mathrm{C} & 2 & 2 & 2.934 & -0.724 & -0.180 \\ \text { HETATM } & 13 & 0 & 2 & 2 & 2.590 & 1.266 & 2.771 \\ \text { HETATM } & 14 & \mathrm{C} & 2 & 2 & 2.182 & 0.887 & 1.751 \\ \text { HETATM } & 15 & 0 & 2 & 2 & 1.865 & 2.892 & -1.062 \\ \text { HETATM } & 16 & \mathrm{C} & 2 & 2 & 1.668 & 1.877 & -0.518 \\ \text { HETATM } & 17 & 0 & 2 & 2 & -3.370 & -2.394 & -0.023 \\ \text { HETATM } & 18 & \mathrm{C} & 2 & 2 & -2.693 & -1.464 & -0.103 \\ \text { HETATM } & 19 & 0 & 2 & 2 & -3.300 & 1.323 & -2.183 \\ \text { HETATM } & 20 & \mathrm{C} & 2 & 2 & -2.693 & 0.826 & -1.339 \\ \text { HETATM } & 21 & 0 & 2 & 2 & -1.074 & 2.683 & 1.123 \\ \text { HETATM } & 22 & \mathrm{C} & 2 & 2 & -1.167 & 1.655 & 0.617 \\ \text { HETATM } & 23 & \mathrm{H} & 2 & 2 & -2.791 & 0.309 & 0.989\end{array}$




$\begin{array}{lrrrrrrr}\begin{array}{l}{[2 \mathrm{H}(\mathrm{g})] 1-} \\ \text { HEADER }\end{array} & & & & & & & \\ \text { HETATM } & 1 & \mathrm{H} & 2 & 2 & -1.542 & 2.693 & -0.546 \\ \text { HETATM } & 2 & \mathrm{H} & 2 & 2 & -0.359 & 3.520 & -1.580 \\ \text { HETATM } & 3 & \mathrm{H} & 2 & 2 & -0.131 & 4.186 & 0.866 \\ \text { HETATM } & 4 & \mathrm{H} & 2 & 2 & 1.356 & 3.448 & 0.182 \\ \text { HETATM } & 5 & \mathrm{C} & 2 & 2 & -0.475 & 2.781 & -0.775 \\ \text { HETATM } & 6 & \mathrm{C} & 2 & 2 & 0.310 & 3.268 & 0.454 \\ \text { HETATM } & 7 & \mathrm{FE} & 2 & 2 & -1.090 & -0.676 & -0.582 \\ \text { HETATM } & 8 & \mathrm{FE} & 2 & 2 & 1.106 & 0.070 & 0.421 \\ \text { HETATM } & 9 & \mathrm{~S} & 2 & 2 & 0.162 & 1.155 & -1.436 \\ \text { HETATM } & 10 & \mathrm{~S} & 2 & 2 & 0.287 & 1.940 & 1.761 \\ \text { HETATM } & 11 & 0 & 2 & 2 & -3.247 & 0.989 & 0.545 \\ \text { HETATM } & 12 & \mathrm{C} & 2 & 2 & -2.368 & 0.347 & 0.129 \\ \text { HETATM } & 13 & 0 & 2 & 2 & -1.800 & -2.978 & 1.059 \\ \text { HETATM } & 14 & \mathrm{C} & 2 & 2 & -1.514 & -2.061 & 0.407 \\ \text { HETATM } & 15 & 0 & 2 & 2 & -2.139 & -1.671 & -3.129 \\ \text { HETATM } & 16 & \mathrm{C} & 2 & 2 & -1.689 & -1.275 & -2.135 \\ \text { HETATM } & 17 & 0 & 2 & 2 & 3.913 & 0.623 & -0.126 \\ \text { HETATM } & 18 & \mathrm{C} & 2 & 2 & 2.787 & 0.434 & 0.104 \\ \text { HETATM } & 19 & 0 & 2 & 2 & 1.314 & -2.420 & -1.065 \\ \text { HETATM } & 20 & \mathrm{C} & 2 & 2 & 0.775 & -1.458 & -0.625 \\ \text { HETATM } & 21 & 0 & 2 & 2 & 1.610 & -1.623 & 2.744 \\ \text { HETATM } & 22 & \mathrm{C} & 2 & 2 & 1.404 & -0.952 & 1.819 \\ \text { HETATM } & 23 & \mathrm{H} & 2 & 2 & 1.369 & 2.415 & 2.446\end{array}$

$\begin{array}{lrrrrrrr}\begin{array}{l}{[2 \mathrm{H}(\mathrm{h})] 1-} \\ \text { HEADER }\end{array} & & & & & & & \\ \text { HETATM } & 1 & \mathrm{H} & 2 & 2 & 1.474 & -2.792 & 0.478 \\ \text { HETATM } & 2 & \mathrm{H} & 2 & 2 & 1.961 & -1.331 & 1.357 \\ \text { HETATM } & 3 & \mathrm{H} & 2 & 2 & 3.904 & -2.687 & 0.472 \\ \text { HETATM } & 4 & \mathrm{C} & 2 & 2 & 1.933 & -1.801 & 0.370 \\ \text { HETATM } & 5 & \mathrm{C} & 2 & 2 & 3.358 & -1.996 & -0.183 \\ \text { HETATM } & 6 & \mathrm{H} & 2 & 2 & 3.313 & -2.419 & -1.194 \\ \text { HETATM } & 7 & \mathrm{FE} & 2 & 2 & 0.098 & 1.232 & 0.046 \\ \text { HETATM } & 8 & \mathrm{FE} & 2 & 2 & -1.386 & -0.841 & -0.059 \\ \text { HETATM } & 9 & \mathrm{~S} & 2 & 2 & 0.847 & -0.839 & -0.799 \\ \text { HETATM } & 10 & \mathrm{~S} & 2 & 2 & 4.294 & -0.385 & -0.240 \\ \text { HETATM } & 11 & 0 & 2 & 2 & 1.443 & 0.942 & 2.672 \\ \text { HETATM } & 12 & \mathrm{C} & 2 & 2 & 0.908 & 1.030 & 1.648 \\ \text { HETATM } & 13 & 0 & 2 & 2 & -0.775 & -2.242 & 2.479 \\ \text { HETATM } & 14 & \mathrm{C} & 2 & 2 & -0.996 & -1.666 & 1.497 \\ \text { HETATM } & 15 & 0 & 2 & 2 & -1.591 & 3.411 & 1.002 \\ \text { HETATM } & 16 & \mathrm{C} & 2 & 2 & -0.919 & 2.547 & 0.624 \\ \text { HETATM } & 17 & 0 & 2 & 2 & -4.028 & -0.002 & 0.856 \\ \text { HETATM } & 18 & \mathrm{C} & 2 & 2 & -2.979 & -0.336 & 0.494 \\ \text { HETATM } & 19 & \mathrm{C} & 2 & 2 & -1.362 & 0.775 & -1.198 \\ \text { HETATM } & 20 & 0 & 2 & 2 & -1.967 & 1.250 & -2.098 \\ \text { HETATM } & 21 & 0 & 2 & 2 & 1.786 & 2.895 & -1.669 \\ \text { HETATM } & 22 & \mathrm{C} & 2 & 2 & 1.111 & 2.223 & -1.010 \\ \text { HETATM } & 23 & 0 & 2 & 2 & -2.317 & -2.857 & -1.960 \\ \text { HETATM } & 24 & \mathrm{C} & 2 & 2 & -1.944 & -2.039 & -1.228 \\ \text { HETATM } & 25 & \mathrm{H} & 2 & 2 & 5.513 & -0.948 & -0.469\end{array}$


axial, basal $[2 \mathrm{~B}+2] 2$ -

\begin{tabular}{|c|c|c|c|c|c|c|c|}
\hline HEADER & & & & & & & \\
\hline HETATM & 1 & $\mathrm{H}$ & 2 & 2 & -4.023 & 3.386 & -0.636 \\
\hline HETATM & 2 & $\mathrm{H}$ & 2 & 2 & -2.675 & 3.009 & -1.734 \\
\hline HETATM & 3 & $\mathrm{H}$ & 2 & 2 & -5.424 & 2.434 & -2.252 \\
\hline HETATM & 4 & $\mathrm{H}$ & 2 & 2 & -4.047 & 1.716 & -3.122 \\
\hline HETATM & 5 & C & 2 & 2 & -3.516 & 2.576 & -1.176 \\
\hline HETATM & 6 & C & 2 & 2 & -4.497 & 1.857 & -2.131 \\
\hline HETATM & 7 & H & 2 & 2 & 1.591 & -0.618 & -1.424 \\
\hline HETATM & 8 & $\mathrm{H}$ & 2 & 2 & 0.415 & 0.378 & -0.531 \\
\hline HETATM & 9 & $\mathrm{H}$ & 2 & 2 & -0.012 & -2.284 & -1.077 \\
\hline HETATM & 10 & C & 2 & 2 & 1.069 & $-\odot .499$ & -0.468 \\
\hline HETATM & 11 & C & 2 & 2 & 0.226 & -1.761 & -0.140 \\
\hline HETATM & 12 & $\mathrm{H}$ & 2 & 2 & 0.816 & -2.457 & $\odot .470$ \\
\hline HETATM & 13 & FE & 2 & 2 & 4.402 & -1.067 & $\odot .246$ \\
\hline HETATM & 14 & FE & 2 & 2 & 3.877 & 1.429 & 0.006 \\
\hline HETATM & 15 & FE & 2 & 2 & -4.731 & 0.340 & 0.786 \\
\hline HETATM & 16 & FE & 2 & 2 & -2.980 & -0.682 & -0.744 \\
\hline HETATM & 17 & $\mathrm{~S}$ & 2 & 2 & 2.339 & -0.125 & 0.847 \\
\hline HETATM & 18 & $\mathrm{~S}$ & 2 & 2 & -2.783 & 1.442 & 0.111 \\
\hline HETATM & 19 & $\mathrm{~S}$ & 2 & 2 & -4.972 & $\odot .156$ & -1.536 \\
\hline HETATM & 20 & $\mathrm{~S}$ & 2 & 2 & -1.357 & -1.464 & $\odot .795$ \\
\hline HETATM & 21 & 0 & 2 & 2 & -6.644 & 2.459 & 1.460 \\
\hline HETATM & 22 & C & 2 & 2 & -5.865 & 1.650 & 1.167 \\
\hline HETATM & 23 & 0 & 2 & 2 & -3.556 & -0.038 & 3.443 \\
\hline HETATM & 24 & C & 2 & 2 & -3.991 & $\odot .099$ & 2.384 \\
\hline HETATM & 25 & 0 & 2 & 2 & -6.372 & -2.067 & 1.083 \\
\hline HETATM & 26 & C & 2 & 2 & -5.702 & -1.129 & 0.956 \\
\hline HETATM & 27 & 0 & 2 & 2 & -1.334 & -0.538 & -3.136 \\
\hline HETATM & 28 & C & 2 & 2 & -1.966 & -0.603 & -2.168 \\
\hline HETATM & 29 & 0 & 2 & 2 & -3.661 & -3.525 & -0.979 \\
\hline HETATM & 30 & C & 2 & 2 & -3.405 & -2.403 & $-\odot .877$ \\
\hline HETATM & 31 & 0 & 2 & 2 & 5.049 & -1.444 & 3.117 \\
\hline HETATM & 32 & C & 2 & 2 & 4.787 & -1.256 & 2.003 \\
\hline HETATM & 33 & 0 & 2 & 2 & 4.254 & 2.501 & 2.748 \\
\hline HETATM & 34 & C & 2 & 2 & 4.112 & 2.048 & 1.691 \\
\hline HETATM & 35 & 0 & 2 & 2 & 7.187 & -1.400 & -0.562 \\
\hline HETATM & 36 & C & 2 & 2 & 6.080 & -1.267 & -0.236 \\
\hline HETATM & 37 & 0 & 2 & 2 & 6.301 & 2.724 & -0.977 \\
\hline HETATM & 38 & C & 2 & 2 & 5.338 & 2.208 & -0.582 \\
\hline HETATM & 39 & C & 2 & 2 & 4.139 & 0.045 & -1.366 \\
\hline HETATM & 40 & 0 & 2 & 2 & 4.185 & $-\odot .063$ & -2.547 \\
\hline HETATM & 41 & 0 & 2 & 2 & 3.459 & -3.640 & -0.760 \\
\hline HETATM & 42 & C & 2 & 2 & 3.810 & -2.601 & -0.378 \\
\hline HETATM & 43 & 0 & 2 & 2 & 1.990 & 3.181 & -1.370 \\
\hline HETATM & 44 & C & 2 & 2 & 2.727 & 2.461 & -0.838 \\
\hline
\end{tabular}

axial, basal $[2 \mathrm{~B}+2] 1-$

$\begin{array}{lrlrrrrr}\text { HEADER } & & & & & & \\ \text { HETATM } & 1 & \mathrm{H} & 2 & 2 & 2.649 & 3.095 & 1.364 \\ \text { HETATM } & 2 & \mathrm{H} & 2 & 2 & 1.776 & 2.092 & 2.542 \\ \text { HETATM } & 3 & \mathrm{H} & 2 & 2 & 4.623 & 2.379 & 2.397 \\ \text { HETATM } & 4 & \mathrm{H} & 2 & 2 & 3.762 & 1.122 & 3.314 \\ \text { HETATM } & 5 & \mathrm{C} & 2 & 2 & 2.545 & 2.077 & 1.759 \\ \text { HETATM } & 6 & \mathrm{C} & 2 & 2 & 3.893 & 1.564 & 2.318 \\ \text { HETATM } & 7 & \mathrm{H} & 2 & 2 & -1.715 & -1.715 & 1.609 \\ \text { HETATM } & 8 & \mathrm{H} & 2 & 2 & -0.292 & -0.670 & 1.330 \\ \text { HETATM } & 9 & \mathrm{H} & 2 & 2 & 0.183 & -3.170 & 1.107 \\ \text { HETATM } & 10 & \mathrm{C} & 2 & 2 & -0.984 & -1.379 & 0.865 \\ \text { HETATM } & 11 & \mathrm{C} & 2 & 2 & -0.180 & -2.554 & 0.272\end{array}$




$\begin{array}{lrrrrrrr}\text { HETATM } & 12 & \mathrm{H} & 2 & 2 & -0.828 & -3.190 & -0.348 \\ \text { HETATM } & 13 & \mathrm{FE} & 2 & 2 & -4.175 & -0.820 & -0.471 \\ \text { HETATM } & 14 & \mathrm{FE} & 2 & 2 & -3.037 & 1.323 & 0.374 \\ \text { HETATM } & 15 & \mathrm{FE} & 2 & 2 & 3.842 & 0.726 & -0.869 \\ \text { HETATM } & 16 & \mathrm{FE} & 2 & 2 & 2.861 & -1.045 & 0.645 \\ \text { HETATM } & 17 & \mathrm{~S} & 2 & 2 & -1.844 & -0.415 & -0.485 \\ \text { HETATM } & 18 & \mathrm{~S} & 2 & 2 & 1.891 & 1.025 & 0.367 \\ \text { HETATM } & 19 & \mathrm{~S} & 2 & 2 & 4.660 & 0.236 & 1.261 \\ \text { HETATM } & 20 & \mathrm{~S} & 2 & 2 & 1.242 & -2.018 & -0.788 \\ \text { HETATM } & 21 & 0 & 2 & 2 & 4.884 & 3.433 & -1.322 \\ \text { HETATM } & 22 & \mathrm{C} & 2 & 2 & 4.454 & 2.378 & -1.116 \\ \text { HETATM } & 23 & 0 & 2 & 2 & 2.236 & 0.478 & -3.310 \\ \text { HETATM } & 24 & \mathrm{C} & 2 & 2 & 2.850 & 0.553 & -2.338 \\ \text { HETATM } & 25 & 0 & 2 & 2 & 5.983 & -0.915 & -2.010 \\ \text { HETATM } & 26 & \mathrm{C} & 2 & 2 & 5.128 & -0.285 & -1.553 \\ \text { HETATM } & 27 & 0 & 2 & 2 & 1.810 & -1.896 & 3.222 \\ \text { HETATM } & 28 & \mathrm{C} & 2 & 2 & 2.218 & -1.567 & 2.187 \\ \text { HETATM } & 29 & 0 & 2 & 2 & 4.422 & -3.470 & 0.105 \\ \text { HETATM } & 30 & \mathrm{C} & 2 & 2 & 3.818 & -2.508 & 0.306 \\ \text { HETATM } & 31 & 0 & 2 & 2 & -4.151 & 0.589 & -3.084 \\ \text { HETATM } & 32 & \mathrm{C} & 2 & 2 & -4.138 & 0.070 & -2.059 \\ \text { HETATM } & 33 & 0 & 2 & 2 & -2.065 & 3.418 & -1.532 \\ \text { HETATM } & 34 & \mathrm{C} & 2 & 2 & -2.450 & 2.611 & -0.814 \\ \text { HETATM } & 35 & 0 & 2 & 2 & -7.086 & -0.471 & -0.364 \\ \text { HETATM } & 36 & \mathrm{C} & 2 & 2 & -5.944 & -0.603 & -0.405 \\ \text { HETATM } & 37 & 0 & 2 & 2 & -5.425 & 2.764 & 1.293 \\ \text { HETATM } & 38 & \mathrm{C} & 2 & 2 & -4.484 & 2.202 & 0.933 \\ \text { HETATM } & 39 & \mathrm{C} & 2 & 2 & -4.145 & -0.969 & 1.334 \\ \text { HETATM } & 40 & 0 & 2 & 2 & -4.201 & -1.150 & 2.472 \\ \text { HETATM } & 41 & 0 & 2 & 2 & -4.035 & -3.633 & -1.328 \\ \text { HETATM } & 42 & \mathrm{C} & 2 & 2 & -4.074 & -2.534 & -0.998 \\ \text { HETATM } & 43 & 0 & 2 & 2 & -1.287 & 2.012 & 2.669 \\ \text { HETATM } & 44 & \mathrm{C} & 2 & 2 & -1.975 & 1.735 & 1.790 \\ & & & & & & & \end{array}$

apical [2D+2]2-

$\begin{array}{lrrrrrrr}\text { HEADER } & & & & & & & \\ \text { HETATM } & 1 & \mathrm{H} & 2 & 2 & -0.852 & -2.706 & 1.209 \\ \text { HETATM } & 2 & \mathrm{H} & 2 & 2 & -2.521 & -3.272 & 1.498 \\ \text { HETATM } & 3 & \mathrm{H} & 2 & 2 & -1.114 & -2.849 & -1.069 \\ \text { HETATM } & 4 & \mathrm{H} & 2 & 2 & -2.713 & -3.609 & -0.811 \\ \text { HETATM } & 5 & \mathrm{C} & 2 & 2 & -1.903 & -2.558 & 0.938 \\ \text { HETATM } & 6 & \mathrm{C} & 2 & 2 & -2.090 & -2.733 & -0.586 \\ \text { HETATM } & 7 & \mathrm{H} & 2 & 2 & -0.150 & -0.549 & -2.954 \\ \text { HETATM } & 8 & \mathrm{H} & 2 & 2 & 0.523 & 1.084 & -2.681 \\ \text { HETATM } & 9 & \mathrm{H} & 2 & 2 & 2.107 & -1.554 & -2.590 \\ \text { HETATM } & 10 & \mathrm{H} & 2 & 2 & 2.223 & -0.287 & -3.838 \\ \text { HETATM } & 11 & \mathrm{C} & 2 & 2 & 0.617 & 0.025 & -2.411 \\ \text { HETATM } & 12 & \mathrm{C} & 2 & 2 & 2.024 & -0.477 & -2.772 \\ \text { HETATM } & 13 & \mathrm{FE} & 2 & 2 & -1.892 & 0.514 & -0.264 \\ \text { HETATM } & 14 & \mathrm{FE} & 2 & 2 & -4.222 & -0.340 & 0.263 \\ \text { HETATM } & 15 & \mathrm{FE} & 2 & 2 & 4.069 & -0.822 & 0.097 \\ \text { HETATM } & 16 & \mathrm{FE} & 2 & 2 & 2.320 & 1.039 & 0.194 \\ \text { HETATM } & 17 & \mathrm{~S} & 2 & 2 & -2.348 & -0.862 & 1.550 \\ \text { HETATM } & 18 & \mathrm{~S} & 2 & 2 & -2.877 & -1.265 & -1.409 \\ \text { HETATM } & 19 & \mathrm{~S} & 2 & 2 & 0.322 & -0.165 & -0.590 \\ \text { HETATM } & 20 & \mathrm{~S} & 2 & 2 & 3.352 & 0.415 & -1.809 \\ \text { HETATM } & 21 & 0 & 2 & 2 & -1.306 & 2.769 & 1.506 \\ \text { HETATM } & 22 & \mathrm{C} & 2 & 2 & -1.521 & 1.872 & 0.815 \\ \text { HETATM } & 23 & 0 & 2 & 2 & -2.158 & 2.281 & -2.574 \\ \text { HETATM } & 24 & \mathrm{C} & 2 & 2 & -2.043 & 1.575 & -1.664 \\ \text { HETATM } & 25 & 0 & 2 & 2 & -4.937 & 1.676 & 2.262\end{array}$




$\begin{array}{lllllrrr}\text { HETATM } & 26 & \mathrm{C} & 2 & 2 & -4.639 & 0.887 & 1.468 \\ \text { HETATM } & 27 & 0 & 2 & 2 & -5.719 & 1.058 & -1.829 \\ \text { HETATM } & 28 & \mathrm{C} & 2 & 2 & -5.113 & 0.515 & -1.003 \\ \text { HETATM } & 29 & 0 & 2 & 2 & -6.096 & -2.497 & 0.927 \\ \text { HETATM } & 30 & \mathrm{C} & 2 & 2 & -5.326 & -1.671 & 0.663 \\ \text { HETATM } & 31 & 0 & 2 & 2 & 2.601 & -3.284 & -0.594 \\ \text { HETATM } & 32 & \mathrm{C} & 2 & 2 & 3.138 & -2.286 & -0.318 \\ \text { HETATM } & 33 & 0 & 2 & 2 & 4.418 & -1.602 & 2.883 \\ \text { HETATM } & 34 & \mathrm{C} & 2 & 2 & 4.267 & -1.282 & 1.772 \\ \text { HETATM } & 35 & 0 & 2 & 2 & 6.839 & -1.233 & -0.749 \\ \text { HETATM } & 36 & \mathrm{C} & 2 & 2 & 5.737 & -1.026 & -0.421 \\ \text { HETATM } & 37 & 0 & 2 & 2 & 1.851 & 3.854 & -0.374 \\ \text { HETATM } & 38 & \mathrm{C} & 2 & 2 & 2.003 & 2.716 & -0.169 \\ \text { HETATM } & 39 & 0 & 2 & 2 & 4.889 & 1.797 & 1.340 \\ \text { HETATM } & 40 & \mathrm{C} & 2 & 2 & 4.092 & 1.098 & 0.793 \\ \text { HETATM } & 41 & 0 & 2 & 2 & 1.393 & 1.093 & 2.960 \\ \text { HETATM } & 42 & \mathrm{C} & 2 & 2 & 1.752 & 1.069 & 1.856\end{array}$

apical [2D+2]1-

\begin{tabular}{|c|c|c|c|c|c|c|c|}
\hline HEADER & & & & & & & \\
\hline HETATM & 1 & $\mathrm{H}$ & 2 & 2 & 1.157 & -2.699 & -1.527 \\
\hline HETATM & 2 & $\mathrm{H}$ & 2 & 2 & 2.847 & -3.117 & -1.906 \\
\hline HETATM & 3 & H & 2 & 2 & 1.513 & -3.221 & $\odot .697$ \\
\hline HETATM & 4 & $\mathrm{H}$ & 2 & 2 & 3.205 & -3.644 & $\odot .341$ \\
\hline HETATM & 5 & C & 2 & 2 & 2.202 & -2.517 & -1.252 \\
\hline HETATM & 6 & C & 2 & 2 & 2.441 & -2.864 & 0.234 \\
\hline HETATM & 7 & $\mathrm{H}$ & 2 & 2 & 0.144 & -1.466 & 2.625 \\
\hline HETATM & 8 & $\mathrm{H}$ & 2 & 2 & -0.389 & $\odot .220$ & 2.814 \\
\hline HETATM & 9 & $\mathrm{H}$ & 2 & 2 & -2.194 & -2.154 & 2.021 \\
\hline HETATM & 10 & $\mathrm{H}$ & 2 & 2 & -2.220 & -1.285 & 3.574 \\
\hline HETATM & 11 & C & 2 & 2 & -0.573 & -0.714 & 2.269 \\
\hline HETATM & 12 & C & 2 & 2 & -2.017 & -1.186 & 2.499 \\
\hline HETATM & 13 & FE & 2 & 2 & 1.913 & 0.394 & 0.275 \\
\hline HETATM & 14 & FE & 2 & 2 & 4.291 & -0.185 & -0.245 \\
\hline HETATM & 15 & FE & 2 & 2 & -4.335 & -0.541 & -0.162 \\
\hline HETATM & 16 & FE & 2 & 2 & -2.201 & 0.774 & -0.118 \\
\hline HETATM & 17 & $\mathrm{~s}$ & 2 & 2 & 2.531 & -0.735 & -1.660 \\
\hline HETATM & 18 & $\mathrm{~s}$ & 2 & 2 & 3.005 & -1.416 & 1.255 \\
\hline HETATM & 19 & $\mathrm{~S}$ & 2 & 2 & -0.262 & -0.442 & $\odot .456$ \\
\hline HETATM & 20 & $\mathrm{~S}$ & 2 & 2 & -3.222 & $\odot .086$ & 1.853 \\
\hline HETATM & 21 & 0 & 2 & 2 & 1.310 & 2.790 & -1.294 \\
\hline HETATM & 22 & C & 2 & 2 & 1.541 & 1.844 & -0.677 \\
\hline HETATM & 23 & 0 & 2 & 2 & 2.025 & 1.916 & 2.770 \\
\hline HETATM & 24 & C & 2 & 2 & 1.978 & 1.311 & 1.787 \\
\hline HETATM & 25 & 0 & 2 & 2 & 4.934 & 2.060 & -2.016 \\
\hline HETATM & 26 & C & 2 & 2 & 4.672 & 1.180 & -1.317 \\
\hline HETATM & 27 & 0 & 2 & 2 & 5.625 & 1.081 & 2.038 \\
\hline HETATM & 28 & C & 2 & 2 & 5.093 & 0.588 & 1.141 \\
\hline HETATM & 29 & 0 & 2 & 2 & 6.353 & -2.110 & -1.067 \\
\hline HETATM & 30 & C & 2 & 2 & 5.531 & -1.367 & -0.742 \\
\hline HETATM & 31 & 0 & 2 & 2 & -2.867 & -3.104 & -0.357 \\
\hline HETATM & 32 & C & 2 & 2 & -3.410 & -2.087 & -0.286 \\
\hline HETATM & 33 & 0 & 2 & 2 & -5.446 & -0.935 & -2.836 \\
\hline HETATM & 34 & C & 2 & 2 & $-5 . \odot \odot 4$ & -0.775 & -1.783 \\
\hline HETATM & 35 & 0 & 2 & 2 & -6.881 & $-\odot .939$ & 1.239 \\
\hline HETATM & 36 & C & 2 & 2 & -5.884 & -0.763 & $\odot .683$ \\
\hline HETATM & 37 & 0 & 2 & 2 & -1.519 & 3.499 & $\odot .819$ \\
\hline HETATM & 38 & C & 2 & 2 & -1.825 & 2.462 & $\odot .420$ \\
\hline HETATM & 39 & 0 & 2 & 2 & -4.736 & 2.306 & -0.908 \\
\hline HETATM & 40 & C & 2 & 2 & -4.224 & 1.317 & -0.551 \\
\hline HETATM & 41 & 0 & 2 & 2 & -1.431 & 1.122 & -2.924 \\
\hline HETATM & 42 & C & 2 & 2 & -1.718 & 0.987 & -1.816 \\
\hline
\end{tabular}

